\title{
Analysis of factors affecting the prognosis of patients with cervical intraepithelial neoplasia 2
}

\author{
XIAOBO ZHANG, YOUGUI XU, TIANYU MENG and DANHUA SHEN \\ Department of Pathology, Peking University People's Hospital, Beijing 100044, P.R. China
}

Received October 1, 2019; Accepted April 6, 2020

DOI: $10.3892 / 01.2020 .11711$

\begin{abstract}
According to the 2014 World Health Organization Classification of Tumors of Female Reproductive Organs, patients with cervical intraepithelial neoplasia 2 (CIN2) have an equivocal diagnosis, but p16 is considered as the reference index for CIN2. Positive p16 expression in CIN2 is associated with high-grade squamous intraepithelial lesions (HSIL), whereas p16 negative lesions are low-grade squamous intraepithelial lesions. The purpose of the present study was to examine the clinical value of p16 and human papillomavirus (HPV) E6/E7 mRNA in the prognostication of patients with CIN2. From January 2013 to January 2016, 108 patients were diagnosed with CIN2 by biopsy and followed up at 6-month intervals at Peking University People's Hospital (Beijing, China). The expression of HPV E6/E7 mRNA was detected by in situ hybridization, while the expression of p16 and $\mathrm{Ki}-67$ proteins was detected by immunohistochemistry. Of the 108 CIN2 cases, 20 progressed to HSIL/CIN3, 36 cases demonstrated persistence with CIN2 after the follow-up and 52 cases achieved regression ( $\leq \mathrm{CIN} 1)$. Of the p16-positive 82 cases, 20 cases were detected to have progressed, whereas in the p16-negative group, no progression was observed. There were statistically significant differences among the p16-positive and negative groups $(\mathrm{P}<0.05)$. In the HPV E6/E7 mRNA-positive 69 cases, 18 cases were detected to have progressed, whereas in the HPV E6/E7 mRNA-negative 39 cases, progression was detected in only 2 cases. There were statistically significant differences among the HPV E6/E7 mRNA-positive and negative groups $(\mathrm{P}<0.05)$. The area under the receiver operating characteristics curve was plotted; the area under the curve for HPV E6/E7 mRNA was 0.745, that for p16 was 0.546 and that for Ki-67 was 0.501 . The detection of HPV E6/E7 mRNA may provide important predictive information for the prognosis of CIN2, however p16 and Ki-67 proteins may provide little value.
\end{abstract}

Correspondence to: Professor Danhua Shen, Department of Pathology, Peking University People's Hospital, 11 Xizhi Men South Street, Xicheng, Beijing 100044, P.R. China

E-mail: danhuashen@126.com

Key words: cervical intraepithelial neoplasia 2, human papillomavirus E6/E7 mRNA, p16, prognosis

\section{Introduction}

Cervical carcinoma is the second most common malignancy among females worldwide. It is preceded by a long precursor phase, characterized by cervical intraepithelial neoplasia (CIN), which may persist for 10-20 years. High-grade CIN is the precursor of cervical cancer. Certain evidence indicated that spontaneous regression occurs in $\sim 40 \%$ of cases of CIN2 (1), suggesting that these lesions probably have characteristics of being less aggressive compared to CIN3 or more severe lesions. The regression rate of CIN2 is similar to that of CIN1 in a 2-year follow-up period (2). At present, histopathological assessment is unable to differentiate high-grade CIN lesions from others and predict whether they may regress spontaneously. Certain prognostic biomarkers may be helpful in this differentiation (3).

Important progress in the etiological study of cervical cancer has been made in the last decade, and it is now widely accepted that high-risk human papillomavirus (HR-HPV) is the central cause of cervical cancer and precursor lesions (4). HPV E6/E7 mRNA, which is translated into E6 and E7 proteins, is able to directly reflect the target of malignant transformation of host cells. Testing HPV E6/E7 mRNA transcription is considered as the gold standard for verifying the presence of HPV. As a novel RNA in situ detection method, the RNAscope in situ hybridization (ISH) method is able to directly detect HPV, specifically in the process of E6/E7 mRNA transcription, thus providing evidence of HPV transcription activity in cells. This method may avoid errors of other indirect methods (5). It has been reported that the sensitivity and specificity of HPV E6/E7 mRNA detected by the RNAscope ISH method is similar to that of p16 and $\mathrm{Ki}-67$ detected by immunohistochemistry (IHC) in head and neck tumors (6). The 2 combination algorithms for the RNAscope HPV test and p16-IHC were reported to be superior to p16-IHC alone in predicting the prognosis of oropharyngeal cancers (7).

In cervical cancer detection, HPV E6/E7 mRNA is useful for the differential diagnosis of challenging low-grade squamous intraepithelial lesion (LSIL) vs. benign reactive change (8). While the role of single factors, e.g. p16 and HPVE6/E7 mRNA, has been demonstrated in previous studies, it remains elusive whether HPV E6/E7 mRNA has a role in the progression of CIN2 lesions. The objective of the present study was to evaluate the usefulness of HPV E6/E7 mRNA and p16 in predicting the evolution of CIN2 in patients 
without prior treatment and to provide an objective basis for guiding clinical treatment.

\section{Materials and methods}

Patient selection and study design. A prospective study was performed from the files of the Department of Pathology at the Peking University People's Hospital (Beijing, China) between March 2013 and March 2016. In total, 108 cases that were histologically confirmed to have CIN2 as the most severe lesion at the time of the initial diagnosis and those who preferred cautious waiting rather than immediate treatment were included in the study. Patients who remained untreated for 6 months or longer after the diagnosis of CIN2 were also included in the study. The exclusion criteria were as follows: Pregnancy at the time of diagnosis, a previous diagnosis of CIN3 or more severe lesions, as well as treatments, including cervical conization, loop electrosurgical excision procedure or hysterectomy, prior to diagnosis (9). This study was approved by the Ethics Committee of Peking University People's Hospital (Beijing, China) and informed consent was waived.

In situ hybridization. HR-HPV RNAscope ISH was performed by using RNAscope ${ }^{\circledR} 2.5$ HD assay-brown to detect HPV E6/E7 mRNA, including HPV type 16, 18, 26, 31, 33, 35, 39, 45, 51, 52, 53, 56, 58, 59, 66, 68, 73 and 82 ('HR RNA18' ISH, cocktail probe). RNAscope assays were performed following the manufacturer's instructions. Ubiquitin controls were performed using HeLa control slides provided by Advanced Cell Diagnostics. After unstained slides were cut, directed punches from formalin-fixed, paraffin-embedded tissues were taken from areas of morphologic correlation with $\mathrm{H} \& \mathrm{E}$ slides. Positive signals were recorded for dark-brown, dot-like cytoplasmic and/or nuclear staining, while a slide with no staining was considered as 'negative' (8). Each case was examined alongside a positive control (cervical squamous cell carcinoma) and a negative control (normal cervical squamous epithelium).

IHC. For p16 ${ }^{\mathrm{INK} 4 \mathrm{a}}$ detection, the CINtec Histology Kit (clone E6H4; Roche Diagnostics) was used following the manufacturer's protocol. IHC was performed with the Ventana Benchmark XT-automatic staining machine. 'Positivity' referred to nuclear and/or cytoplasm staining and was defined as a continuous staining of cells in the basal and para-basal layers, with or without staining of superficial squamous cell layers. A 'negative' slide was defined as having no staining, staining of only isolated cells or small cell clusters, or non-continuous staining (i.e. usually patchy or focal cell pattern) $(10,11)$.

Ki-67 rat anti-human monoclonal antibody (cat. no. EP5; 1:200) was obtained from Beijing Zhongshan Jinqiao Biotechnology Co. Positive Ki-67 scores of 1, 2 and 3 were assigned to samples in which nuclear staining was detectable in $<5,6-25$ and $>25 \%$, respectively, of the epithelial region (excluding basal cells), the IHC of Ki-67 were performed following the manufacturer's instructions (12).

Thin-prep cytologic test (TCT). TCTs were performed by using the Thin-Prep T2000 slide processor (Hologic) and stained using the Papanicolaou method. Cytological diagnoses were made according to the Bethesda System (13).

Pathological evaluation. All of the H\&E-stained slides, p16, Ki-67 immunostaining and HPV E6/E7 mRNA were independently reviewed by 2 pathologists, $\mathrm{XZ}$ and DS blinded to the previous results. H\&E-stained slides and the results for p16, Ki-67 and HPVE6/E7 mRNA were reviewed.

Follow-up. A follow-up visit was performed at least 6 months after the initial CIN2 diagnosis. At the follow-up visit, cervical samples were processed for Pap tests and HR-HPV testing. Certain patients underwent colposcopy and cervical biopsy. The progression of cervical lesions was defined as follows $(14,15)$. Progression: High-grade squamous intraepithelial lesion (HSIL)/CIN3 or squamous cell carcinoma by histological diagnosis, except for adenocarcinoma in situ or cervical adenocarcinoma. Regression: A lesion with a histological diagnosis lower than CIN2, including negative cytologic or biopsy findings, LSIL or atypical squamous cells of undetermined significance as the most severe diagnosis after follow-up. Persistence: Histological CIN2 was diagnosed.

Statistical analysis. The data were analyzed by SPSS statistics v25.0 software (IBM Corp.). The positive and negative proportions of p16 and HPV E6/E7 mRNA were determined among the cases. The association of positive HPV E6/E7 mRNA and positive p16 with subsequent CIN3 were analyzed using Fisher's exact tests. Receiver operating characteristic (ROC) curves were plotted with GraphPad Prism 8.0 software (GraphPad Software, Inc.) and areas under the curve (AUCs) were determined for further assessment of the prognostic value. $\mathrm{P}<0.05$ was considered to indicate statistical significance and predict prognosis of CIN2.

\section{Results}

Clinicopathological characteristics. In total, 108 cases with biopsy specimens diagnosed as CIN2 and available follow-up data were included. The patients' age ranged from 19 to 66 years, with a mean age of 46.3 years. Follow-up intervals ranged from 6 to 36 months, with a mean of 26.3 months and a median of 20 months.

Of the 108 cases with CIN2 status, 20 cases progressed to HSIL/CIN3. Patients who progressed to CIN3 were immediately treated by cone resection. In none of the cases, progression to invasive squamous cell carcinoma was observed. There were 36 cases with persistence of CIN2 after follow-up and 52 cases exhibited regression to grades $\leq$ LSIL/CIN1. This was at the individual end of follow-up for each patient. Representative images of IHC detection of E6/E7 mRNA are displayed in Fig. 1 and those for p16 and $\mathrm{Ki}-67$ protein are provided in Fig. 2.

p16 ${ }^{I N K 4 a}$ and Ki-67 HPV E6/E7 mRNA expression and influence on the prognosis of CIN2. Among the 108 CIN2 cases, the positive rate of p16 expression was $75.9 \%(82 / 108)$ and the negative rate of p16 was $24.1 \%$ (26/108; Table I). Of the p16-positive cases, 20 cases were detected to have progression, 28 cases had persistence and 34 cases had regression at the 

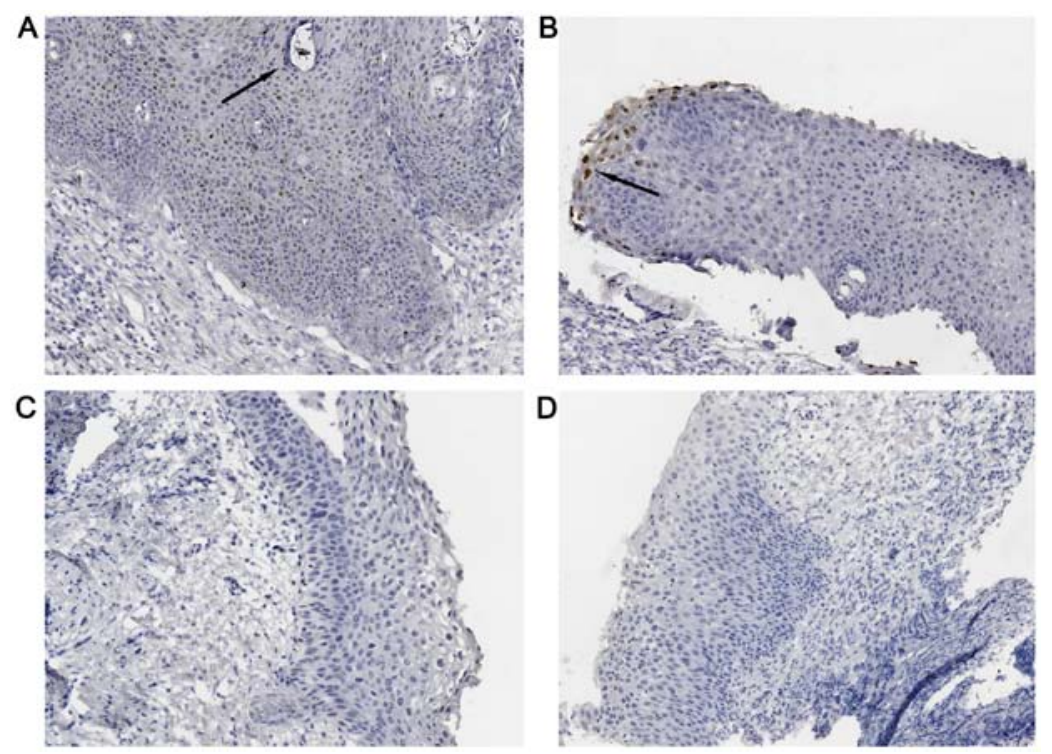

Figure 1. Representative immunohistochemistry images for detecting HPV E6/E7 mRNA in cervical intraepithelial neoplasia 2 tissues. (A and B) HPV E6/E7 mRNA-positive samples; dots and clusters with positive staining were observed in cervical squamous epithelial cells (arrows; magnification, x200). (C and D) HPV E6/E7 mRNA-negative samples with no staining in cervical squamous epithelial cells (magnification, x200). HPV, human papillomavirus.
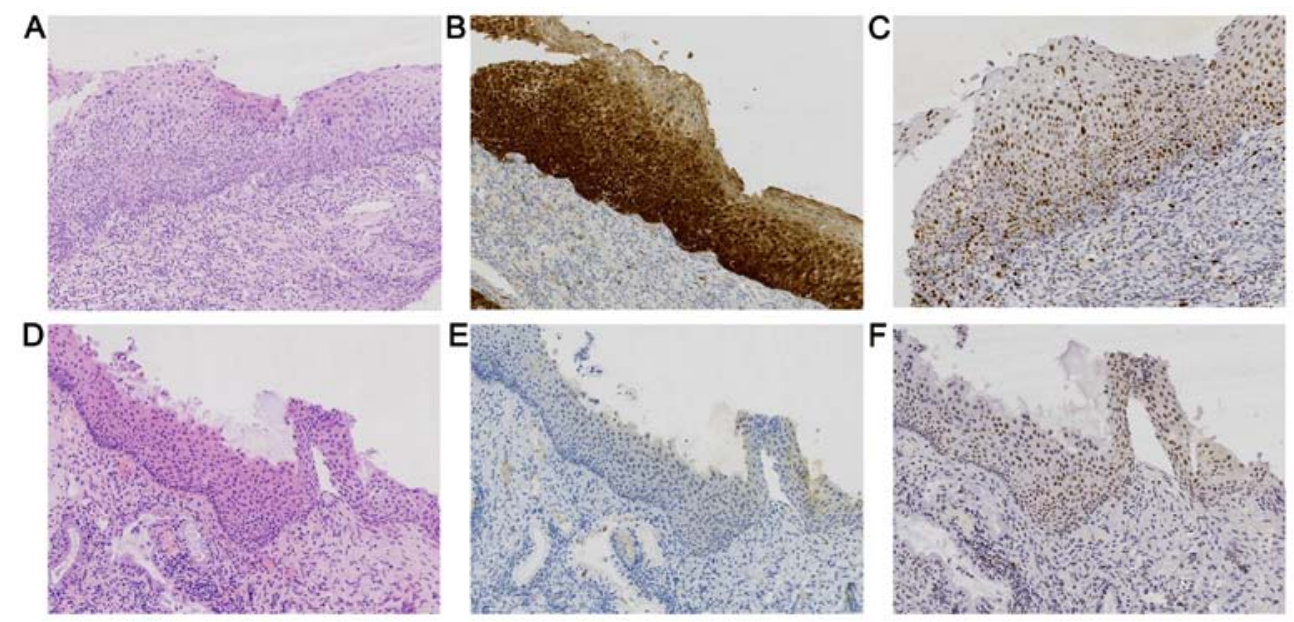

Figure 2. Representative immunohistochemistry images for detecting p16 and Ki-67 in CIN2 tissues. (A) CIN2, H\&E stain. (B) Positive for p16. (C) Ki-67 expression in $>25 \%$ of cells. (D) CIN2, H\&E stain. (E) negative p16 stain. (F) Ki-67 expression in $>25 \%$ of cells. (D-F, another case) (magnification, x200 for all). CIN2, cervical intraepithelial neoplasia 2.

individual end of follow-up for each patient. In the p16-negative group, no progression occurred in any of the patients, 8 cases had persistence and 18 cases exhibited regression. There were statistically significant differences among these groups as indicated by Fisher's test $(\mathrm{P}<0.05)$. For predicting progression, the sensitivity of p16 was $100 \%(20 / 20+0)$ and the specificity was $34.6 \%(18 / 18+34$; Table II).

The positive rate of HPV E6/E7 mRNA expression was $63.9 \%(69 / 108)$ and the negative rate was $36.1 \%$ (39/108; Table I). In the HPV E6/E7 mRNA-positive group, 18 cases were determined to have progression, 19 cases had persistence and 32 cases exhibited regression. In the HPV E6/E7 mRNA-negative group, 2 cases were detected to have progression, 17 cases had persistence and 20 cases exhibited regression. There were statistically significant differences among these groups according to Fisher's test $(\mathrm{P}<0.05)$. For predicting progression, the sensitivity of HPV E6/E7 mRNA was $90.0 \%(18 / 18+2)$ and the specificity was $38.5 \%(20 / 20+32$; Table II).

For 59/108 cases, p16 and HPV E6/E7 mRNA were positive in the same patient. The rate of progression to HSIL/CIN3 for p16+/HPV E6/E7 mRNA+ cases was 16.7\% (18/108). For predicting progression, the sensitivity and specificity of p16+/HPV E6/E7 mRNA+ was 90.0 and 34.2\%, respectively (Table II).

Regarding staining for Ki-67, none of the eight cases with $<5 \% \mathrm{Ki}-67$ was detected to have progression, while $5 / 54$ cases in the $6-25 \% \mathrm{Ki}-67$ group and $15 / 46$ cases in the $>25 \%$ Ki-67 group exhibited progression. There were statistically significant differences among the groups $(\mathrm{P}<0.05)$.

According to the association of HPV E6/E7 mRNA, P16, Ki-67 with the progression to CIN3 results, the ROCs were plotted. The AUC of HPV E6/E7 mRNA, P16 and Ki-67 was $0.745,0.546$ and 0.501 , respectively (Fig. 3). 
Table I. Association of different variables with the prognosis of CIN2.

\begin{tabular}{|c|c|c|c|c|c|}
\hline \multirow[b]{2}{*}{ Feature } & \multirow[b]{2}{*}{ Total } & \multicolumn{3}{|c|}{ Prognosis of CIN2 } & \multirow[b]{2}{*}{ P-value } \\
\hline & & Progression & Persistence & Regression & \\
\hline p16 & & & & & 0.002 \\
\hline$(+)$ & $82(75.9)$ & $20(18.5)$ & $28(25.9)$ & $34(31.5)$ & \\
\hline$(-)$ & $26(24.1)$ & $0(0)$ & $8(7.4)$ & $18(16.7)$ & \\
\hline Ki-67 staining (\%) & & & & & 0.027 \\
\hline$<5$ & $8(7.4)$ & $0(0)$ & $2(1.8)$ & $6(5.6)$ & \\
\hline $6-25$ & $54(50.0)$ & $5(4.6)$ & $20(18.5)$ & $29(26.9)$ & \\
\hline$>25$ & $46(42.6)$ & $15(13.9)$ & $14(13.0)$ & $17(15.7)$ & \\
\hline HPVE6/E7 & & & & & 0.014 \\
\hline$(+)$ & $69(63.9)$ & $18(16.7)$ & $19(17.6)$ & $32(29.6)$ & \\
\hline$(-)$ & $39(36.1)$ & $2(1.9)$ & $17(15.7)$ & $20(18.5)$ & \\
\hline Gland involvement & & & & & 0.191 \\
\hline Yes & $14(13.0)$ & $6(5.6)$ & $5(4.6)$ & $3(2.8)$ & \\
\hline No & $94(87.0)$ & $14(13.0)$ & $31(28.7)$ & $49(45.3)$ & \\
\hline Age (years) & & & & & 0.082 \\
\hline$\leq 30$ & $27(25.0)$ & $6(5.6)$ & $9(8.3)$ & $12(11.1)$ & \\
\hline$>30$ & $81(75.0)$ & $14(13.0)$ & $27(25.0)$ & $40(37.0)$ & \\
\hline
\end{tabular}

Values are expressed as n (\%). CIN2, cervical intraepithelial neoplasia 2; HPV, human papillomavirus.

Table II. Value of p16 and HPVE6/E7 expression and association with subsequent CIN3.

\begin{tabular}{lccccrr}
\hline $\begin{array}{l}\text { Expression } \\
\text { status }\end{array}$ & $\begin{array}{c}\text { Total } \\
\text { positive cases }\end{array}$ & Subsequent CIN3 & Sensitivity (\%) & Specificity (\%) & \multicolumn{3}{c}{$\begin{array}{c}\text { Positive predictive Negative predictive } \\
\text { value }(\%)\end{array}$} \\
\hline P16+ & $82(75.9)$ & $20(18.5)$ & 100 & 34.6 & 37.0 & 100.0 \\
HPVE6/E7+ & $69(63.9)$ & $18(16.7)$ & 90.0 & 38.5 & 36.0 & 90.9 \\
P16+HPVE6/E7+ & $59(54.6)$ & $18(16.7)$ & 90.0 & 34.2 & 41.9 & 100.0 \\
\hline
\end{tabular}

Values are expressed as n (\%) unless otherwise indicated. CIN3, cervical intraepithelial neoplasia 3; HPV, human papillomavirus.

Influence of age and gland involvement on the prognosis of patients with CIN2. All 108 cases were divided into 2 groups based on age. Of the patients aged $\leq 30$ years, 6/27 cases exhibited progression, and among those aged $>30$ years, $14 / 81$ cases had progression ( $\mathrm{P}>0.05$ according to Fisher's test). Furthermore, 14 cases had gland involvement, while 94 cases had no gland involvement $(\mathrm{P}>0.05)$.

\section{Discussion}

The name and grading system of CIN has been officially formalized since 2003 (16). However, this naming system has been controversial, as the diagnostic repeatability for CIN2 is poor and there inevitably exists inter-observer variability, resulting in numerous CIN2 lesions that are frequently misclassified (over- or underdiagnosed). CIN2 represents an unclear biological entity and is an admixture of transient lesions and true pre-cancer lesions (10). In 2012, the American Society of Pathologists and the American Society of Colposcopy and Cervical Pathology generated a report from the Lower Anogenital Squamous Terminology (LAST) project, suggesting that cervical lesions should be divided into LSIL and HSIL (10). This system was also endorsed by the 2014 World Health Organization Classification of Tumors of Female Reproductive Organs (17). Among them, CIN1 was included in LSIL, CIN3 was included in HSIL and for the morphologically equivocal CIN2, p16 was considered as the reference index. CIN2 with a p16-positive status belongs to HSIL and p16-negative lesions, formerly classified as CIN2, may be downgraded to LSIL to simplify clinical management (10).

The management of CIN2 is still a problem for gynecologists. In the past, certain studies have demonstrated that CIN2 has spontaneously regressed in young females. The results of the present study are similar to those of most published reports (14), with a $48.1 \%$ spontaneous regression rate of CIN2 during the least 6 months of follow-up in patients, independent of their age. Therefore, a conservative follow-up approach may 


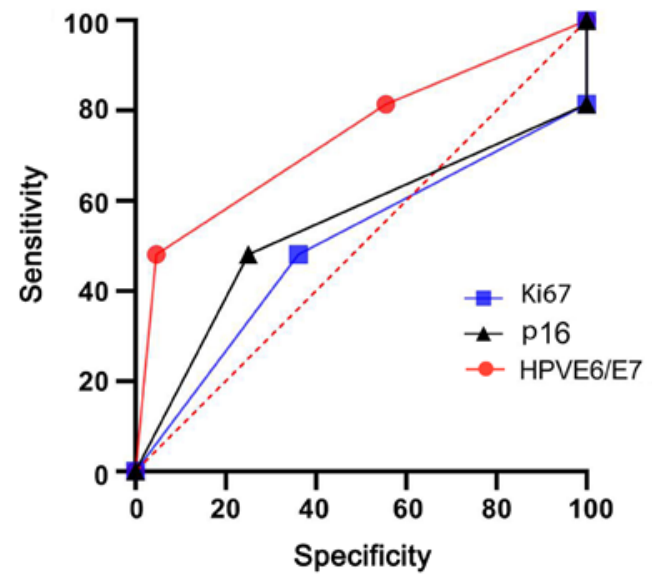

Figure 3. Receiver operating characteristic curve of HPV E6/E7 mRNA, p16 and $\mathrm{Ki}-67$ in progression to CIN3. CIN 3, cervical intraepithelial neoplasia 3; HPV, human papillomavirus.

be considered to reduce unnecessary cone excision for these patients.

For the diagnosis of cervical lesions, p16 is an important marker. In the present study, the positive rate of $\mathrm{p} 16$ was $75.9 \%(82 / 108)$, whereas the negative rate was $24.1 \%$ (27/108). Of the p16-positive cases, 20 cases were detected to have progression; however, in the negative group, no progression occurred. There were statistically significant differences among these groups $(\mathrm{P}<0.05)$. The group with p16-negative CIN2 biopsies only had regression or persistence but no progression. Conversely, CIN2 cases positive for p16 were at a higher risk of evolving into HSIL/CIN3 lesions. The sensitivity of p16 was $100 \%$, the specificity was $34.6 \%$, the positive predictive value was $37 \%$ and the negative predictive value was $100 \%$. These results indicate that expression levels of p16 are closely associated with the outcome of CIN2 lesions and support the idea that p16 is able to identify cases more likely to have definitive pre-cancer features at the time of follow-up $(15,18,19)$.

The sensitivity of p16 for predicting future progression to CIN3 was the highest (100\%), demonstrating that p16 positive may progress to HSIL. When the negative predictive value of p16 was the highest (100\%), a negative status for p16 only led to regression or persistence and did not lead to progression. The result of the present study was consistent with the recommendation of the LAST study (20). Another study exploring the value of p16 immunostaining for improving the diagnostic accuracy in cervical biopsy tissues indicated that p16 was more sensitive and less specific in diagnosing lesions as CIN2 or worse as compared with routine histopathological assessments (21). However, this may not be the best way to choose a cutoff value where the sensitivity is $100 \%$. This is a limitation of the present study.

The following two utilities have been recognized for p16: First, p16 may be used to distinguish benign or reactive changes from precursor lesions associated with HR-HPV (19). Second, p16 may be used to separate LSIL from HSIL. The results are consistent with those of several studies $(12,22,23)$ that have evaluated the role of p16 as a predictive marker for the outcome of LSIL (20-22). In general, lesions with LSIL status with diffuse positive p16 staining had a significantly higher tendency to progress to high-grade lesions than p16-negative lesions.

Although p16-positive CIN2 lesions exhibited a significantly higher tendency to persist or progress than p16-negative lesions, $31.5 \%$ (34/108) of p16-positive CIN2 lesions had regressed at follow-up. If all p16-positive CIN2 lesions are considered as high-grade lesions, this may lead to overtreatment of patients in this group. For p16-negative cases, the primary approach should be regular observation with follow-up. However, it has been reported that a small percentage of p16-negative CIN2 lesions progress to CIN3, so additional diagnostic and prognostic markers may still be required (24).

HPV E6/E7, which are translated into E6 and E7 proteins, may directly reflect the malignant transformation information of host cells and serve as an important biomarker. As one of the early coding products in the HPV genome after HPV infection, E6 and E7 proteins react with human tumor suppressor gene products, p53 and retinoblastoma protein (pRb), respectively, and block the p53-dependent apoptotic pathway, leading to carcinogenesis (24). HR-HPV E6/E7 mRNA is an indicator of the active state of the HPV oncogene and the expression of HR-HPV E6/E7 mRNA indicates that the virus has started the process of integration and carcinogenesis, leading to the occurrence of HSIL. It may be a good assessment factor for monitoring HPV infection and predicting the progression of lesions to more severe cervical lesions and even cervical cancer. HPV E6/E7 mRNA has been suggested as a novel screening method for cervical lesions (25). The identification of HPV mRNA is of particular clinical appeal, as it is well established that transcriptionally active HR-HPV with dysregulated expression of oncogenes E6 and E7 is necessary for neoplastic transformation (26).

In the present study, the positive rate of HPV E6/E7 mRNA was $63.9 \%(69 / 108)$, whereas the negative rate was $36.1 \%$ (39/108) in CIN2 lesions. This positive rate is lower than that reported in a previous study (8). In the HPV E6/E7 mRNA-positive group, 18 cases were detected to have progression, whereas in the negative group, only 2 cases were detected to have progression. The differences in expression levels of HPV E6/E7 mRNA were statistically significant among the different prognosis groups of CIN2 lesions $(\mathrm{P}<0.05)$. The progression rate in the HPV E6/E7 mRNA-positive group was higher than that in the negative group. This indicates that HPV E6/E7 mRNA may be used as a clinical predictive marker to identify the risk of developing more aggressive lesions from CIN2. A prospective study that used liquid-based cervical cytology samples collected over 24 months reported that $91 \%$ of cases with an LSIL cytological result and E6/E7-positive status were detected as having progressed, while $85 \%$ of E6/E7-negative cases had regressed (27). For predicting progression, the sensitivity of HPV E6/E7 mRNA was $90 \%$ and the specificity was $38.5 \%$, whereas the positive predictive value and negative predictive value was 36 and $90.9 \%$, respectively. The sensitivity of HPV E6/E7 mRNA for predicting future CIN3 was high (up to 90\%). HPV E6/E7 mRNA-positive patients may have an increased risk of progression to HSIL, whereas patients with negative predictive values (up to $90.9 \%$ ) may be more likely to have regression or persistence, while having a lower risk of progression. HPV E6/E7 mRNA was more sensitive and 
less specific for predicting the outcome of CIN2 but performed worse than routine histopathological assessments. This result is similar to the study by Frega et al (28), where HPV E6/E7 mRNA was indicated to have a high sensitivity and negative predictive value, which may be used to predict the recurrence of cervical lesions.

Studies have indicated that the expression level of HPVE6/E7 is correlated with the severity of cervical lesions. As the lesion grade increased, the positive rate of E6/E7 mRNA exhibited an increasing trend (29). Methylation assays (as a surrogate marker for the accumulation of genetic and epigenetic changes as a result of persisting effects of HPV E6/E7 mRNA expression in high-grade CIN) are highly efficient at detecting advanced CIN lesions (30). HPV E6/E7 mRNA may have an important role in the diagnostic evaluation and prediction of prognosis, but at present, HPV E6/E7 mRNA as a predictive marker to identify the risk of developing more aggressive lesions in CIN2 has been rarely reported.

In the present study, 59 of the 108 cases were positive for p16 and HPV E6/E7 combined. The sensitivities of P16+, HPVE6/E7+ and P16+HPVE6/E7+ status for predicting future CIN3 were similar (100\% vs. $90 \%$ vs. $90 \%)$. The specificity of the three indicators was also similar $(34.6 \%$ vs. $38.5 \%$ vs. $34.2 \%$ ), as were the positive predictive value and the negative predictive value. The simultaneous presence of HPV E6/E7 mRNA and p16 protein may reflect the regulation of p16 by HPV E6/E7 in the development of cervical cancer and pre-cancerous lesions. When HPV virus is capable of transcriptional activity, $\mathrm{pRb}$ in HPV-positive cells, in combination with HPVE7 protein, may render E2F transcription factor continuously activated. This process leads to the failure of $\mathrm{pRb}$ to combine with p16 protein, resulting in excessive deposition and overexpression of p16 protein in cells (8).

$\mathrm{Ki}-67$ is a nuclear antigen associated with cell proliferation and its expression is associated with the pathogenesis of pre-cancerous lesions. In the present study, a statistically significant association of higher expression of Ki-67 with a higher risk of disease progression was observed $(\mathrm{P}<0.05)$. For instance, none of the cases with $<5 \% \mathrm{Ki}-67$ staining progressed, while $15 / 46$ of the cases with $>25 \% \mathrm{Ki}-67$ staining progressed. This result is similar to that reported by Baak et al (31).

When the ROC curves of HPVE6/E7 mRNA, p16 and Ki-67 were evaluated, the AUC of HPV E6/E7 mRNA, p16 and $\mathrm{Ki}-67$ protein was $0.745,0.546$ and 0.501 , respectively. Of the three factors, the AUC of HPVE6/E7 was the largest with the highest prognostic value for the progression of CIN2, and the AUC of p16 and Ki67 was relatively lower. Of note, only indicators with an AUC of $>0.5$ may be considered to be of sufficient prognostic value, while the two indicators p16 and Ki67 were not sufficient for prognostic evaluation. This is consistent with the suggestion that HPVE6/E7 mRNA may ultimately be superior to p16 in the identification of HR-HPV infection (8). It is also consistent with another study that reported that the two combination algorithms of the HPVE6/E7 mRNA detection and p16 detection were superior to those of p16 alone in predicting prognosis (7).

In an earlier study, Loopik et al (9) suggested that the regressive likelihood is greater in females aged $<25$ years, suggesting a conservative approach option for managing CIN2. A large systematic review and meta-analysis by Tainio et al (14) suggested that there were higher rates of regression and lower rates of progression of histologically confirmed CIN2 lesions, particularly in females aged $\leq 30$. These studies indicated that in younger patients, HPV may be eliminated by the immune system over a certain period. However, the present study was not in accordance with the above-mentioned ones. When the cohort of the present study was stratified by age, there were no significant differences between ages in terms of outcome of CIN2 ( $>0.05)$. The biopsy resection may have stimulated an immune response and altered the course of the disease. The follow-up time was relatively short. The range of lesions may be small and limited. The treatment of CIN2 lesions includes follow-up and treatment. Based on the design of the present study, only patients with follow-up data were selected. Thus, the question as to whether age should be a predictive indicator requires further investigation.

Using gland involvement as a predictive marker to identify the risk of developing more aggressive lesions in patients with CIN2 status has been suggested. In the present study, 14 cases had gland involvement and 94 cases had no glands involvement. There were no statistically significant differences between patients with and without gland involvement in terms of progression $(\mathrm{P}>0.05)$. Thus, in the present study, no association between gland involvement and prognosis of CIN2 lesions was determined, suggesting that it may not serve as a predictive marker.

Of note, the present study had certain limitations. First, it was a prospective study and only untreated cases of CIN2 were reviewed, which may have resulted in a certain degree of bias in the experimental methods. Further studies with a larger number of patients are required to confirm the present results.

The detection of HPV E6/E7 mRNA may provide important predictive information for the prognosis of CIN2, however p16 and Ki-67 proteins alone may provide little value.

\section{Acknowledgements}

The authors would like to thank Dr James Byrd, Department of Gastroenterology, Hepatology and Nutrition Anderson Cancer Center, Houston, TX, USA for his assistance with manuscript editing.

\section{Funding}

No funding was received.

\section{Availability of data and materials}

All data generated or analyzed during the present study are included in this published article.

\section{Authors' contributions}

$\mathrm{XZ}, \mathrm{YX}, \mathrm{TM}$ and DS were involved in performing experiments. YX prepared the materials and XZ contributed to data acquisition and drafted and wrote the manuscript. DS designed and supervised the study. All authors have read and approved the final manuscript. 


\section{Ethics approval and consent to participate}

This study was approved by the Ethics Committee of Peking University People's Hospital (Beijing, China) and informed consent was waived.

\section{Patient consent for publication}

Not applicable.

\section{Competing interests}

The authors declare that they have no competing interests.

\section{References}

1. Christoph G, Stephan P, Camilla N, Rahhal J, Hefler L, Tempfer CB, Heinze G, Stary G, Reinthaller A and Speiser P: Treatment of cervical intraepithelial neoplasia with topical imiquimod: A randomized controlled trial. Obstet Gynecol 120: $152-159,2012$.

2. Aps G, Mag G and Da-Silva VD: Evaluation of Telomerase (hTert), Ki67 and p16ink4a expressions in low and high-grade cervical intraepithelial lesions. Rev Col Bras Cir 44: 131-139, 2017 (In English, Portuguese).

3. Koeneman MM, Roy Fpm K, Nijman HW, Brigitte Fm S, Toon VG and Arnold-Jan K: Natural history of high-grade cervical intraepithelial neoplasia: A review of prognostic biomarkers. Expert Rev Mol Diagn 15: 527, 2015.

4. Xu QX and Zhang ZY: High-risk Human papillomavirus genotypes in cervical lesions and vaccination challenges in China. Asian Pac J Cancer Prev 16: 2193-2197, 2015.

5. Evans MF, Peng Z, Clark KM, Adamson CSC, Ma XJ, Wu X, Wang $\mathrm{H}$, Luo $\mathrm{Y}$ and Cooper K: HPV E6/E7 RNA in situ hybridization signal patterns as biomarkers of three-tier cervical intraepithelial neoplasia grade. PLoS One 9: e91142, 2014.

6. Mirghani H, Casiraghi O, Amen F, He M, Ma XJ, Saulnier P, Lacroix L, Drusch F, Lakdhar A, Saint Guily JL, et al: Diagnosis of HPV-driven head and neck cancer with a single test in routine clinical practice. Mod Pathol 28: 1518-1527, 2015.

7. Mirghani H, Casiraghi O, Guerlain J, Amen F, He MX, Ma XJ, Luo Y, Mourareau C, Drusch F, Lakdhar AB, et al: Diagnosis of HPV driven oropharyngeal cancers: Comparing p16 based algorithms with the RNAscope HPV-test. Oral Oncology 62: 101-108, 2016

8. Mills AM, Dirks DC, Poulter MD, Mills SE and Stoler MH: HR-HPV E6/E7 mRNA in situ hybridization: Validation against PCR, DNA in situ hybridization, and p16 immunohistochemistry in 102 samples of cervical, vulvar, anal, and head and neck Neoplasia. Am J Surg Pathol 41: 607-615, 2017.

9. Loopik DL, Doucette S, Bekkers RL and Bentley JR: Regression and progression predictors of CIN2 in women younger than 25 years. J Low Genit Tract Dis 20: 213-217, 2016.

10. Darragh TM, Colgan TJ, Cox TJ, Heller DS, Henry MR, Luff RD, McCalmont T, Nayar R, Palefsky JM, Stoler MH, et al: The lower anogenital squamous terminology standardization project for HPV-associated lesions: Background and consensus recommendations from the College of American Pathologists and the American Society for Colposcopy and Cervical Pathology. Int J Gynecol Pathol 32: 76-115, 2013.

11. Christine B, Guglielmo R, Miriam R, Nicolas W, Marc A, Mark S and Magnus von Knebel D: The clinical impact of using p16(INK4a) immunochemistry in cervical histopathology and cytology: An update of recent developments. Int J Cancer 136: $2741-2751,2015$

12. Xiaobo Z and Danhua S: p16INK4a and Ki-67 measurement predict progression of cervical low-grade squamous intraepithelial lesion. Int J Clin Exp Pathol 11: 4109-4116, 2018.

13. Wilbur DC and Nayar R: The Bethesda system for reporting cervical cytology: A historical perspectiv. Acta Cytologica 61: 359-372, 2017.
14. Tainio K, Athanasiou A, Tikkinen KAO, Aaltonen R, Cárdenas J, Hernándes, Glazer-Livson S, Jakobsson M, Joronen K, Kiviharju M, et al: Clinical course of untreated cervical intraepithelial neoplasia grade 2 under active surveillance: Systematic review and meta-analysis. BMJ 360: k499, 2018.

15. Miralpeix E, Genovés J, Maria SSJ, Mancebo G, Lloveras B, Bellosillo B, Alameda F and Carreras R: Usefulness of p16 ${ }^{\mathrm{INK}} 4 \mathrm{a}$ staining for managing histological high-grade squamous intraepithelial cervical lesions. Mod Pathol 30: 304-310, 2016.

16. Tavassoéli FA and Devilee P: Pathology and genetics of tumours of the breast and female genital organs. Lyon, World Health Organization Classification of Tumors, pp432, 2003.

17. Kurman RJ, Carcangiu ML, Herrington CS and Young RH: WHO classification of tumous of female reproductive organs. Fourth edition, pp307, 2014.

18. Miyamoto S, Hasegawa J, Morioka M, Hirota Y, Kushima M and Sekizawa A: The association between p16 and Ki-67 immunohistostaining and the progression of cervical intraepithelial neoplasia grade 2. Int J Gynaecol Obstet 134: 45-48, 2016.

19. Maniar KP, Sanchez B, Paintal A, Gursel DB and Nayar R: Role of the biomarker p16 in downgrading-IN 2 diagnoses and predicting higher-grade lesions. Am J Surg Pathol 39: 1708-1718, 2015.

20. Nuño T and García F: The lower anogenital squamous terminology project and its implications for clinical care. Obstet Gynecol Clin North Am 40: 225-233, 2013.

21. Galgano MT, Castle PE, Atkins KA, Brix WK, Nassau SR and Stoler MH: Using biomarkers as objective standards in the diagnosis of cervical biopsies. Am J Surg Pathol 34: 1077-1087, 2010.

22. Guang-Dong L, Sellors JW, Hai-Kui S, Xun Z, Yan-Ping B, Jose J, Wen C, Fang-Hui Z, Yan S, Zhi C, et al: p16INK4A immunohistochemical staining and predictive value for progression of cervical intraepithelial neoplasia grade 1: A prospective study in China. Int J Cancer 134: 1715-1724, 2014.

23. Maryam R, Andrée S, Oligny LL, Patey N, Dormoy-Raclet V, Ducruet $\mathrm{T}$ and Bouron-Dal Soglio D: Assessment of correlation between p16INK4a staining, specific subtype of human papillomavirus, and progression of LSIL/CIN1 lesions: First comparative study. Am J Clin Pathol 142: 104-110, 2014.

24. Sun L, Zhang L, Krigman HR and Hagemann IS: p16 immunohistochemistry is not always required for accurate diagnosis of grade 2 squamous intraepithelial lesions. J Low Genit Tract Dis 22: 104-109, 2018.

25. Fan Y and Shen Z: The clinical value of HPV E6/E7 and STAT3 mRNA detection in cervical cancer screening. Pathol Res Pract 214: 767-775, 2018.

26. Gernot H, Mahmood M, Kerstin ID, Pischinger, Thomas WR, Christian FS, Kubista E and Czerwenka KF: Physical state and expression of HPV DNA in benign and dysplastic cervical tissue: Different levels of viral integration are correlated with lesion grade. Gynecol Oncol 92: 873-880, 2004.

27. Holm R, Kraus I, Skomedal H, Langerød A, Kristensen GB and Lyng H: Human papillomavirus DNA and e6/e7 mRNA status in relation to survival of patients treated for cervical squamous cell carcinoma. Open Virol J 2: 74-81, 2008.

28. Frega A, Sesti F, Lombardi D, Votano S, Sopracordevole F, Catalano A, Milazzo GN, Lombardo R, Assorgi C, Olivola S, et al: Assessment of HPV-mRNA test to predict recurrent disease in patients previously treated for CIN 2/3. J Clin Virol 60: 39-43, 2014.

29. Coquillard G, Palao B and Patterson BK: Quantification of intracellular HPV E6/E7 mRNA expression increases the specificity and positive predictive value of cervical cancer screening compared to HPV DNA. Gynecol Oncol 120: 89-93, 2011.

30. Steenbergen RDM, Snijders PJF, Heideman DLAM and Meijer CJLM: Clinical implications of (Epi)genetic changes in HPV-induced cervical precancerous lesions. Nat Rev Cancer 14: 395-405, 2014

31. Baak JPA, Arnold-Jan K, Garland SM, Skaland I, Janssen EAM, Tabrizi S, Fagerheim S, Robboy S and Nilsen ST: Combined p53 and retinoblastoma protein detection identifies persistent and regressive cervical high-grade squamous intraepithelial lesions. Am J Surg Pathol 29: 1062-1066, 2005.

This work is licensed under a Creative Commons Attribution-NonCommercial-NoDerivatives 4.0 International (CC BY-NC-ND 4.0) License. 